This is an author-created, un-copyedited version of an article originally published in Plasma Physics and Controlled Fusion. IOP Publishing Ltd is not responsible for any errors or omissions in this version of the manuscript or any version derived from it. The Version of Record is available online at http://dx.doi.org/10.1088/0741$3335 / 55 / 1 / 014010$. 


\title{
Schemes of microwave heating of overdense plasmas in the stellarator TJ-K
}

\author{
A Köhn ${ }^{1}$, G Birkenmeier ${ }^{2}$, A Chusov ${ }^{1}$, P Diez $^{1}$, A Feuer ${ }^{3}$, \\ U Höfel $^{1}$, H Höhnle ${ }^{1}$, E Holzhauer ${ }^{1}$, W Kasparek ${ }^{1}$, \\ S Merli ${ }^{1}$, M Ramisch ${ }^{1}$, J Seifert ${ }^{1}$, S Wolf ${ }^{1}$ and U Stroth ${ }^{1,2}$ \\ ${ }^{1}$ Institut für Plasmaforschung, Universität Stuttgart, 70569 Stuttgart, Germany \\ ${ }^{2}$ Max-Planck-Institut für Plasmaphysik, EURATOM Association, Garching, \\ Germany \\ ${ }^{3}$ Institut für Strahlwerkzeuge, Universität Stuttgart, 70569 Stuttgart, Germany \\ E-mail: koehn@ipf.uni-stuttgart.de
}

\begin{abstract}
Microwave heating of overdense low-temperature plasmas, whose density exceeds the cutoff density of the injected microwave, is described for the stellarator TJ-K. Three different methods featuring each their own characteristics are briefly presented. It is shown that TJ-K allows both fusion relevant scenarios like heating by electron Bernstein waves and fundamental wave-plasma interactions like parametric decay instabilities to be investigated.
\end{abstract}

PACS numbers: 52.25.Xz, 52.35.Hr, 52.35.Mw, 52.50.Sw, 52.55.Hc, 52.70.Ds, 52.70.Gw, $52.70 . \mathrm{Kz}$

Submitted to: Plasma Phys. Control. Fusion

\section{Introduction}

Microwave heating is a widely-used method in plasma physics. It suffers, however, from the fact that the propagation of microwaves is limited to densities below the cutoff density. In addition, in low-temperature plasmas heating at the electron cyclotron resonance frequency (ECRF) cannot be used as opposed to hot-temperature plasmas, since it requires electron temperatures of $T_{e} \geq 1 \mathrm{keV}$ to be efficient [I]]. In this work, three alternative methods of microwave heating of overdense low-temperature plasmas are presented for the stellarator TJ-K. These are: (i) heating at the upperhybrid resonance (UHR) with $2.45 \mathrm{GHz}$, (ii) heating with electron Bernstein waves (EBWs) at the ECRF and its harmonics by injection of microwaves with $8 \mathrm{GHz}$ and (iii) non-resonant heating with $2.45 \mathrm{GHz}$ below the ECRF.

This paper is organized as follows: The stellarator TJ-K is described in Sec. ㄱ. . UHR heating and the observation of a parametric decay instability is reported in Sec. [3. Section $\mathbb{0}$ describes EBW heating and current drive at TJ-K. Non-resonant heating with microwaves of $2.45 \mathrm{GHz}$ being below the ECRF is described in Sec. 5 . The summary in Sec. [ concludes this paper. 


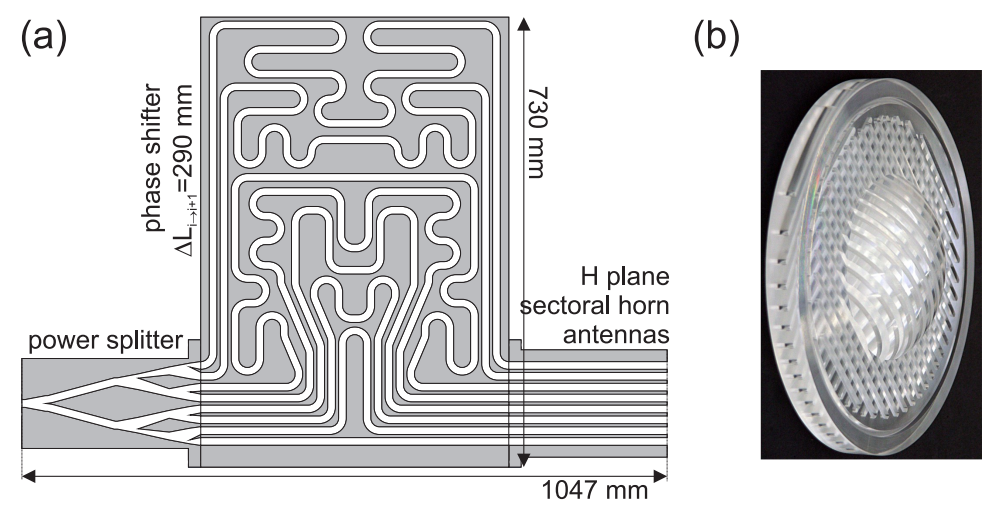

Figure 1. (a) Phased array antenna and (b) numerically optimized lens for the $8 \mathrm{GHz}$ microwave heating system.

\section{The stellarator TJ-K}

TJ-K is an $l=1, m=6$ torsatron with major and minor radii of 0.6 and $0.1 \mathrm{~m}$, respectively [2, 3]. Typical plasma parameters are electron temperatures of $T_{e} \approx 10 \mathrm{eV}$ and plasma densities of $n_{e} \approx 10^{18} \mathrm{~m}^{-3}$. The background magnetic field can be continuously ramped up to a maximum value of $B_{0}=400 \mathrm{mT}$.

\subsection{Microwave heating hardware}

Two microwave heating systems are installed at TJ-K. The first one is based on a magnetron which produces microwaves at $2.45 \mathrm{GHz}$ with a maximum power of $3 \mathrm{~kW}$. The vacuum transition is realized by a quartz window and a simple horn antenna emits the microwaves. The second microwave system consists of a klystron amplifying microwaves in the frequency range $7.9-8.4 \mathrm{GHz}$ to a maximum power of $3 \mathrm{~kW}$. A phased array antenna, as shown in Fig. Wa, is used for emitting the microwaves: the power is split up into 8 waveguides, which act as delay lines and feed $8 \mathrm{H}$-plane sectoral horn antennas. A frequency change results in a variation of the phase difference between the waveguides and thus in a different injection angle. A variation from 7.9 to $8.4 \mathrm{GHz}$ corresponds to an angle variation of $\pm 45^{\circ}$. As a vacuum transition, a lens made of PMMA is used (see Fig. $b$ ). It focuses the microwave beam onto the predicted position of the O-X mode conversion layer, which has been shown to be important for high conversion efficiencies [4]. The final shape of the lens has been defined in an optimization process with the full-wave code IPF-FDMC [4, 55]. Grooves on both sides of the lens minimize microwave reflection [6]. The phased array antenna is installed such that its angle variation is in toroidal direction. Since this corresponds mainly to the direction of the magnetic field, an $\mathrm{O}$-wave is incident into to plasma.

\subsection{Diagnostics}

To obtain the line-averaged density, a Mach-Zehnder interferometer operating at $64 \mathrm{GHz}$ is used [2]. Langmuir probes can be used for measuring density and temperature profiles and for direct investigation of plasma turbulence [ $[\mathbf{Z}, \mathbf{8}]$. A monopole antenna is used to acquire frequency spectra around $2.45 \mathrm{GHz}$. It was previously used to measure wave electric fields [9]. To acquire low frequency spectra 
(a)

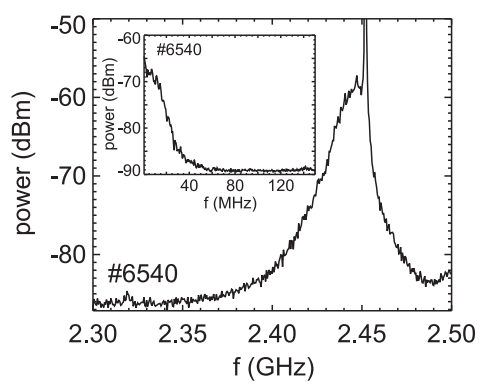

(b)

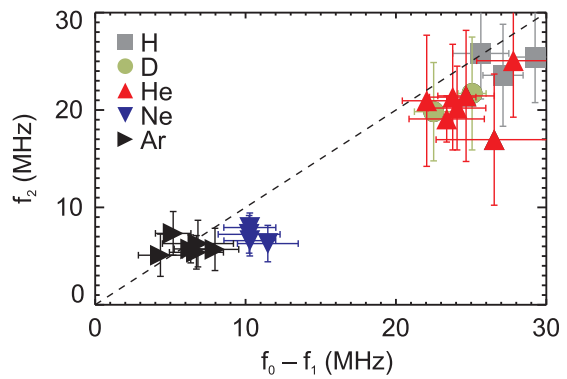

Figure 2. (a) Spectra obtained with a monopole antenna (large plot) and with a loop antenna (small plot) during a helium discharge. (b) Comparison of the spectral width at a decay of $-10 \mathrm{~dB}$ of the high-frequency spectrum (horizontal axis) and the low-frequency spectrum (vertical axis) for different gases.

up to $100 \mathrm{MHz}$, a loop antenna made of stainless steel with a diameter of approximately $37 \mathrm{~mm}$ is used. A similar antenna was successfully used at the W7-AS stellarator to detect lower-hybrid waves [10].

An eight channel bolometer system designed at IPP Garching [II] is used at TJ-K to measure the radiation power losses of the plasma. An external Rogowski coil has been constructed for measuring toroidal net currents. The plasma energy content is obtained from an internal diamagnetic loop.

\section{Heating at the upper-hybrid resonance}

Heating at the UHR is observed at TJ-K with $2.45 \mathrm{GHz}$ in highly overdense plasmas at a magnetic field strength of $B_{0} \approx 70 \mathrm{mT}$, i.e. close to the corresponding ECRF. A maximum of the wave electric field was measured at the UHR [9]. These high values of the wave electric field can give rise to nonlinear phenomena, like parametric decay instabilities [[2, [13]. The simplest case of such an instability is the three wave coupling in which a pump wave with the frequency $f_{0}$ excites two waves with the frequency $f_{1}$ and $f_{2}$. According to the Manley-Rowe relations [14, [5], the frequency and wavenumber matching conditions must be fulfilled: $f_{0}=f_{1}+f_{2}$ and $k_{0}=k_{1}+k_{2}$. Figure 『a shows frequency spectra obtained in a helium discharge. The incident microwave at $f_{0}=2.45 \mathrm{GHz}$ can be clearly identified. A broad sideband on the low-frequency side of $f_{0}$ with a continuous decay down to the edge frequency of $f_{1} \approx 2.4 \mathrm{GHz}$ can be recognized, which corresponds to the high-frequency decay spectrum. In the low-frequency spectrum the corresponding component $f_{2}=f_{0}-f_{1}$ is found. In Fig. [ $2 \mathrm{~b}$, the width of the low-frequency spectrum at a decay of $-10 \mathrm{~dB}$ is plotted as a function of the corresponding high-frequency spectral width for varying ion masses. Both values match well. The scaling with the ion mass, clearly visible in Fig. Z Zlb, points to two candidates in the low-frequency regime: lower-hybrid waves and ion acoustic waves. For the plasma parameters at TJ-K, both waves can exist in the observed frequency regime [I6] and it is not possible to exclude one. It seems likely that both are involved in the parametric decay process. From energy conservation $\left(f_{0}=f_{1}+f_{2}\right)$, it follows that a maximum power of $20 \mathrm{~W}$ can be transferred into the low-frequency component. This power can contribute to ion heating. 

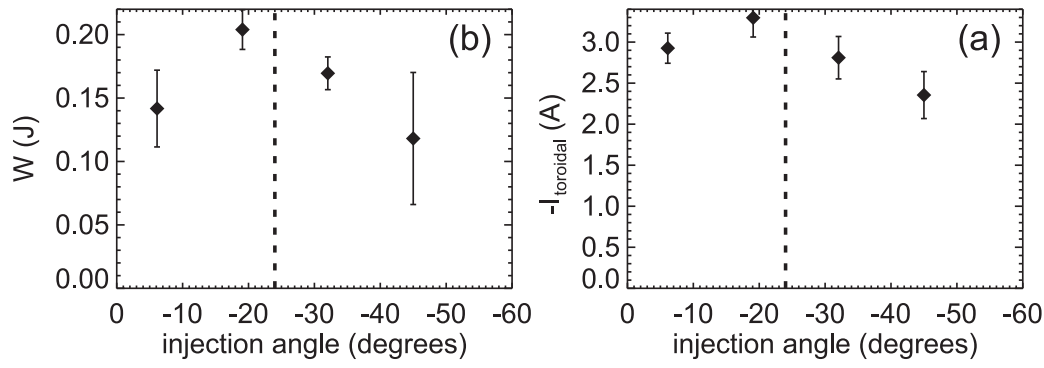

Figure 3. (a) Diamagnetic energy content of the plasma and (b) toroidal net current as a function of the injection angle obtained from a series of helium discharges heated with $8 \mathrm{GHz}$, where the ECRF was located inside the plasma.
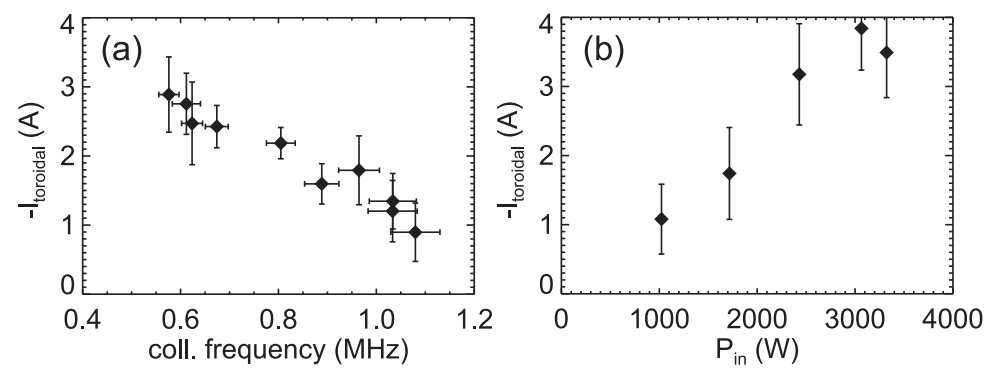

Figure 4. (a) Toroidal net current as a function of the electron-ion collision frequency and (b) as a function of the injected microwave power obtained from a series of helium discharges heated with $8 \mathrm{GHz}$, where the ECRF was located inside the plasma.

\section{Heating with electron Bernstein waves}

EBWs are electrostatic waves and they are absorbed almost completely at the ECRF and its harmonics in high- and low-temperature plasmas [17]. One possibility to excite them is the O-X-B mode conversion process: an injected O-mode is converted to an X-mode around the O-mode cutoff and then into a backwards propagating EBW at the UHR. The efficiency of the whole process depends strongly on the efficiency of the $\mathrm{O}-\mathrm{X}$ conversion which itself depends on the injection angle of the microwave beam with respect to the background magnetic field. Deviations from the optimum angle result in reduced efficiencies, where the degradation is smaller for steeper profiles [5]. The steepness can be characterized by the normalized density scale length $k_{0} L_{n}=k_{0} \cdot n /|\nabla n|$ in the mode conversion region.

At TJ-K, heating with EBWs at the ECRF can be achieved in discharges heated with microwaves around $8 \mathrm{GHz}(3 \mathrm{~kW})$ at $B_{0} \approx 270 \mathrm{mT}$. In these discharges, $k_{0} L_{n} \approx 8$. Figure 5 la shows the energy content of the plasma as a function of the injection angle of the microwave, obtained during a series of helium discharges with $B_{0} \approx 270 \mathrm{mT}$. Each point represents an average over multiple discharges. The energy content of the plasma clearly depends on the injection angle, having its maximum around the calculated optimum value, as indicated by the dashed line. Thus, these measurements represent a strong indication for successful mode conversion and subsequent EBW heating at the fundamental ECRF.

With the Rogowski coil, a toroidal net current on the order of a few amperes 


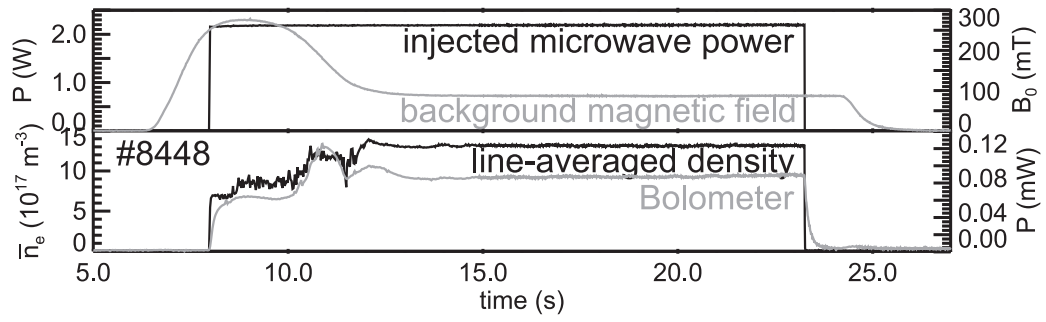

Figure 5. Discharge with heating at harmonics of the ECRF by EBWs.

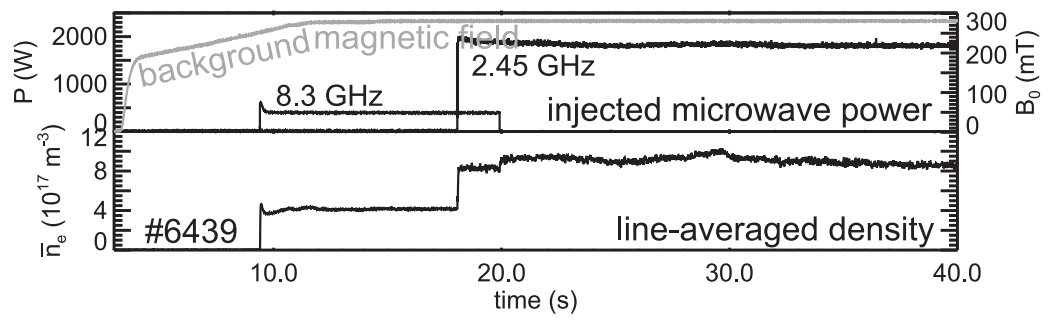

Figure 6. Non-resonantly heated argon discharge.

flowing into counter- $B_{0}$ direction has been detected in this regime. As shown in Fig. [3b, the same angle dependence as for the energy content is found. Two mechanisms can be responsible for current drive by EBWs [ $[\mathbf{Z}]$, the Ohkawa and the Fisch-Boozer mechanism, which is more likely to apply here [I.T]. The expected scaling [20] of the current density $J$ with the electron-ion collision frequency $\nu_{e i}$ and the absorbed power $P_{\text {abs }}, J \propto \nu_{e i}^{-1} P_{\text {abs, }}$, was confirmed experimentally. Figure đla shows the current as a function of $\nu_{e i}$, which was varied by changing the neutral gas pressure in a series of helium discharges. The decrease of the current with increasing collision frequency can clearly be seen. In Fig. $⿴ 囗 十)$, the current is shown as a function of the injected microwave power and an increase of the current with increasing power is also clearly

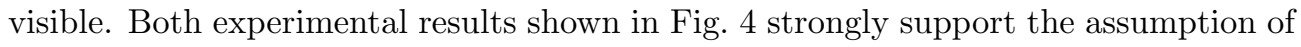
an EBW driven current by the Fisch-Boozer mechanism.

The EBW experiments presented so far were performed with the fundamental ECRF located inside the plasma. Recently, EBW heating at high harmonics of the ECRF has been achieved in TJ-K. A time trace of such a discharge is shown in Fig. 回. First, $B_{0}$ is ramped up and the plasma is started. Then $B_{0}$ is ramped down to $B_{0} \approx 100 \mathrm{mT}$, where the $4^{\text {th }}$ harmonic is situated close to the plasma center. With decreasing $B_{0}$, the density starts to increase, reaching its highest values at the lowest value of $B_{0} \approx 100 \mathrm{mT}$. The bolometer in contrast, has its highest value around $B_{0} \approx 160 \mathrm{mT}$, where the density is strongly fluctuating. Interestingly, for $B_{0} \approx 100 \mathrm{mT}$, the signal of the line-averaged density shows almost no fluctuations, pointing to a very stable plasma. Indeed, using local Langmuir probe measurements, the density fluctuation level has been verified to be as low as a few percent, only.

\section{Heating below the electron cyclotron resonance frequency}

To achieve non-resonant heating at TJ-K, the plasma has to be started resonantly with $8 \mathrm{GHz}$ at $B_{0} \approx 280 \mathrm{mT}$, where there are no resonances for $2.45 \mathrm{GHz}$ inside the 
plasma. Nevertheless, when microwaves at $2.45 \mathrm{GHz}$ are injected, additional heating is observed as shown in Fig. 6. A strong increase in the density can clearly be seen at the moment when the $2.45 \mathrm{GHz}$ is switched on. After the $8 \mathrm{GHz}$ heating is switched off, the density stays at its high level.

The $2.45 \mathrm{GHz}$ is well below the ECRF over the whole plasma cross section and also well below the plasma frequency. Since it is still above the lower-hybrid resonance frequency, we suggest that the heating is due to absorption of Whistler waves inside the plasma. These waves can be absorbed at the so-called O-resonance inside the plasma $[2 \mathrm{ZL}]$. Possible absorption mechanisms are collisional damping and Landau damping. The Whistler waves must be excited via a conversion processes at the plasma boundary [22]. At the moment, further investigations, including full-wave simulations, are performed.

\section{Summary}

Three different schemes of generating overdense low-temperature plasmas have been briefly described for the stellarator TJ-K. It has been shown experimentally that heating at the UHR is accompanied by a parametric decay instability, which can in principle contribute to ion heating. After appropriate shaping of the microwave beam, heating at the ECRF by EBWs and subsequent current drive was found. High density plasmas with very little fluctuations could be produced by high-harmonic heating of EBWs. Finally, an interesting plasma regime, which is heated non-resonantly and characterized by highly overdense plasmas, was presented. In summary, the stellarator TJ-K has proven a device that allows to study both fusion relevant heating scenarios as well as fundamental microwave-plasma interactions.

\section{References}

[1] Bornatici M, Cano R, De Barbieri O, and Engelmann F 1983 Nucl. Fusion 233 |15.3

[2] Krause N et al 2002 Rev. Sci. Instrum. 73:3474

[3] Stroth U et al 2004 Phys. Plasmas 11 25.5

[4] Köhn A et al 2008 Plasma Phys. Control. Fusion 50 (185018

[5] Köhn A et al 2011 Phys. Plasmas 18 18:2501]

[6] Petelin M I, and Kasparek W 1991 Int. Journal of Electronics 71871

[7] Ramisch M et al 2010 Plasma Phys. Control. Fusion 52 1241115

[8] Birkenmeier G, Ramisch M, Manz P, Nold B, and Stroth U 2011 Phys. Rev. Lett. 1117 (1250111

[9] Köhn A et al 2010 Plasma Phys. Control. Fusion 521335003

[10] Suvorov E V et al 1998 Nucl. Fusion 38 661

[11] Mast K F et al 1991 Rev. Sci. Instrum. $\mathbf{6 2} 744$

[12] Yadav V K, and Bora D 2004 Phys. Plasmas 11 34019

[13] Lin A T, and Lin C-C 1981 Phys. Rev. Lett. 47.98

[14] Manley J M, and Rowe H 1956 Proc. IRE 44904

[15] Rowe H 1958 Proc. IRE $46 \times 551$

[16] Stix T H 1992 Waves in plasmas (American Institute of Physics, New York, 1992)

[17] Laqua H P 2007 Plasma Phys. Control. Fusion 4.9 K.1

[18] Urban J et al 2011 Nucl. Fusion 51 18.30bu

[19] Preinhaelter J et al 2009 Plasma Phys. Control. Fusion 51 I25008

[20] Hansen F R, Lynov J P, and Michelsen P 1985 Plasma Phys. Control. Fusion 271077

[21] Heald M A, and Wharton C B 1965 Plasma Diagnostics with Microwaves (John Wiley \& Sons Inc., New York, 1965)

[22] Golant V E, and Piliya A D 1972 Sov. Phys. 14413 